\title{
Orígenes fetales de la patología del adulto: la restricción del crecimiento intrauterino como factor de riesgo
}

\author{
Saulo Molina Giraldo MSc, Diana Marcela Correa Mendoza, José Luis Rojas Arias, \\ Edgar Acuña Osorio.
}

Unidad de Terapia, Cirugía Fetal y Fetoscopia, División de Medicina Materno Fetal, Departamento de Obstetricia y Ginecología, Hospital de San José y Departamento de Ginecología y Obstetricia, Fundación Universitaria de Ciencias de la Salud - FUCS. Bogotá, Colombia.

\section{RESUMEN}

Antecedentes: Barker formuló la hipótesis que la restricción del crecimiento intrauterino (RCIU) representaba un factor de riesgo para el desarrollo de enfermedades durante la vida adulta. Objetivo: Conocer la existencia de alguna relación entre las enfermedades cardiovasculares y enfermedades endocrinas del adulto con el antecedente de RCIU mediante la revisión de estudios publicados de enero de 1990 a diciembre de 2013. Método: Se efectuó una revisión de la literatura existente en las bases de datos PubMed, MEDLINE, ProQuest y Ebsco. Se incluyeron investigaciones en español, inglés o portugués. Resultados: Se incluyeron siete investigaciones uno de casos y controles y seis de cohortes. Para diabetes dos de tres estudios mostraron una asociación significativa (RR=2,10; IC95\% 1,29-3,41; RR=1,43 IC95\% 0,53-3,87; $\mathrm{HR}=1,64 ; \mathrm{IC} 95 \%$ 1,26-2,14). Para hipertensión, dos no observaron relación estadísticamente significativa (RR=0,95; IC95\% 0,81-1,11 y RR=1,03; IC 95\% 0,71-1,49). Para enfermedad coronaria uno mostró asociación significativa (HR=1,9; IC95\% 1,0-3,8). Para obesidad, un estudio no observó relación (Hombres, RR=1,07; IC95\% 0,63-2,29 y mujeres RR=1,07; IC95\% 0,53-2,14). Para síndrome metabólico un estudio observó asociación significativa ( $R R=1,29$; IC95\% 1,00-1,68). Conclusiones: RCIU muestra una relación inconsistente con enfermedades adultas en estudios observaciones que implican seguimiento. Se necesitan estudios longitudinales que corroboren la asociación entre RCIU y enfermedades en la vida adulta observada en algunos análisis previos.

\section{PALABRAS CLAVE: Recién nacido de bajo peso, restricción del crecimiento intrauterino, diabetes, hipertensión, obesidad, programación fetal}

\section{SUMMARY}

Background: Barker hypothesized that intrauterine growth restriction (IUGR) represents a risk factor for developing diseases during adulthood. Objective: To determine the relationship between IUGR and cardiovascular and endocrine diseases in adults reviewing studies published between January 1990 and December 2013. Method: A review of the literature in PubMed, MEDLINE, ProQuest and Ebsco database. Papers in Spanish, English or Portuguese were included. Results: Seven studies were included, one case-control and six cohort design. For diabetes two of three studies showed a significant association (RR=2.10, $95 \% \mathrm{Cl}$ 1.29-3.41; RR=1.43, 95\% Cl 0.53-3.87; $\mathrm{HR}=1.64,95 \% \mathrm{Cl} 1.26-2.14)$ For hypertension, two found no statistically significant (RR=0.95, 95\% $\mathrm{Cl} 0.81-1.11$ and $\mathrm{RR}=1.03,95 \% \mathrm{Cl} 0.71-1.49)$. For coronary disease one showed significant association ( $\mathrm{HR}=1.9,95 \% \mathrm{Cl} 1.0$ to 3.8). For obesity, a study found no relationship (men, $\mathrm{RR}=1.07,95 \% \mathrm{Cl}$ 0.63-2.29; and women $\mathrm{RR}=1.07,95 \% \mathrm{Cl} 0.53-2.14$ ). For metabolic syndrome one 
study found significant association $(\mathrm{RR}=1.29,95 \% \mathrm{Cl} 1.00-1.68)$. Conclusions: IUGR shows an inconsistent relationship with adult diseases in follow up observational researches. Longitudinal studies that corroborate the association between IUGR and adult diseases observed in some previous analyzes are needed.

\section{KEY WORDS: Infant low birth weight, intrauterine growth restriction, diabetes, hypertension, obesity, fetal programming}

\section{INTRODUCCIÓN}

Barker planteó que la Restricción del Crecimiento Intrauterino (RCIU) representaba un factor de riesgo para enfermedades durante la adultez $(1,2)$. RCIU es el peso al nacimiento inferior al percentil 10 con relación a la edad gestacional (3). La desnutrición materna, la insuficiencia placentaria, la exposición a tóxicos y otras circunstancias que disminuyen la oxigenación fetal se asocian a RCIU (4). La 'predisposición' para enfermedades adultas inducida por RCIU se llama 'programación fetal' (5).

A la fecha se ha estudiado la asociación de RCIU y del peso al nacer con enfermedades cardiovasculares (hipertensión arterial) y metabólicas (diabetes, obesidad) (6-9). Barker postuló que el feto que presenta desnutrición manifiesta un fenotipo 'ahorrador' que se expresa en alteraciones en la regulación homeostática, con incremento del consumo de calorías, acúmulo de grasa y la reducción en la producción de energía. Este exceso de calorías disponibles aumenta el riesgo de obesidad y otras condiciones relacionadas con el síndrome metabólico en la adultez $(1,2)$.

La prevalencia de RCIU varía según la definición, la curva de crecimiento de referencia y las características de la población. RCIU afecta cerca del $10 \%$ de los recién nacidos vivos (4). Asimismo, la frecuencia de diabetes, hipertensión arterial y obesidad en adultos muestran un patrón cambiante según las características de las personas. Estas prevalencias se estiman de 2 a $40 \%$ para diabetes, 5 a 58\%, para hipertensión, y 6 a $36 \%$, para obesidad (10-14). En 2008, Whincup y cols en una revisión sistemática mostraron que el peso al nacer se relacionó inversamente con el riesgo de diabetes mellitus tipo 2 en la adultez; la razón de disparidad (OR), ajustada por edad y sexo, fue 0,75 (IC95\% $0,70-0,81)$ por kilo al nacer (15). Sin embargo, en el contexto mundial no se cuenta con una revisión sistemática que resuma la información acerca de la asociación entre RCIU y diabetes, hipertensión y obesidad (16).

El objetivo de este trabajo fue conocer la existencia de alguna relación entre las enfermedades cardiovasculares y enfermedades endocrinas del adulto con el antecedente de restricción del crecimiento intrauterino, mediante la revisión de estudios publicados de enero de 1990 a diciembre de 2013.

\section{MATERIALES Y MÉTODOS}

Para responder la pregunta implícita en el objetivo general se llevó a cabo una revisión de la literatura en las bases de datos PubMed, MEDLINE (Literatura Internacional en Ciencias de la Salud), ProQuest, Ebsco. Se incluyeron investigaciones en español, inglés o portugués, publicadas entre enero de 1990 y diciembre de 2013. Se incluyeron artículos originales, cohortes y casos y controles. Estudios de revisión y presentación de casos se tomaron para la construcción del marco teórico. Se excluyeron revisiones sistemáticas y meta-análisis de investigaciones precedentes. Se complementó la búsqueda en la Biblioteca Virtual en Salud - BIREME (BVS) con la inclusión de LILACS (Literatura Latinoamericana y del Caribe en Ciencias de la Salud), IBECS (Índice Bibliográfico Español en Ciencias de la Salud), Biblioteca Cochrane y SciELO (Scientific Electronic Library Online). La búsqueda de investigaciones se completó mediante la revisión de las referencias de los estudios incluidos.

Se usaron como palabras de búsqueda aquellas relacionadas en el área de ciencias de la salud (DeCS), en español, inglés (Medical Subject Heading, $\mathrm{MeSH}$ ) y portugués: peso fetal (fetal weight; peso fetal), recién nacido de bajo peso (infant low birth weight; recém-nascido de baixo peso), diabetes, hipertensión (hypertension; hipertensão) y obesidad (obesity; obesidade), estudios de casos y controles (case-control studies; estudos de casos e controles) y estudios de cohortes (cohort studies; estudios de cohortes). Adicionalmente, se usó para la búsqueda el término no DeCS restricción de crecimiento intrauterino (Fetal intrauterine growth restriction, restrição do crescimento intrauterino do feto) y hambruna prenatal (prenatal famine; fome pré-natal).

Los autores realizaron la búsqueda electrónica independientemente, revisaron títulos y resúmenes. Posteriormente, los dos autores principales (SM; DC) enfatizaron en el método de las investigaciones y las asociaciones cuantificadas. A los trabajos que llenaron satisfactoriamente esta información se revisaron en texto completo. La revisión incluyó completar una ficha con las características de la población, la definición de RCIU, los desenlaces medidos (enfermedad cardiovascular o metabólica), la frecuencia de RCIU y la medida 
de asociación: razón de disparidad (OR), riesgo relativo $(\mathrm{RR})$ o razón de riesgo $(\mathrm{HR})$, con intervalos de confianza del 95\% (IC95\%). Este análisis implicó una valoración cualitativa.

\section{RESULTADOS}

Se identificaron 152 artículos, discriminados así: 85 relacionados con hipertensión arterial; 54 con diabetes, y 13 con obesidad. De ese grupo se seleccionaron 24 títulos. La lectura del resumen permitió seleccionar 7 artículos para el análisis del texto completo (17-23). Se excluyeron 13 artículos que informaron desenlaces intermedios (tolerancia a la glucosa, medidas antropométricas, etc.) (2436) y 4 que evaluaron desenlaces en menores de edad (37-40).

No se encontraron estudios observacionales longitudinales entre los años 1990 y 2001. Seis de los siete artículos incluidos siguieron un diseño de cohortes $(17,19-23)$ y uno de casos y controles (18). Dos de los artículos informaron diferentes desenlaces de la misma cohorte $(19,20)$. El desenlace que se informó con mayor frecuencia fue diabetes en tres artículos $(18,21,22)$, dos para hipertensión arterial $(17,23)$ y un artículo cada uno para enfermedad coronaria (19), obesidad (23) y síndrome metabólico (20) (Tabla I).

\section{DIABETES}

En Estados Unidos, Burke y cols (2004) en un estudio de casos y controles anidados en una cohorte (los autores omitieron información de participantes en cada grupo), tomaron como casos los individuos que tuvieron bajo peso al nacer (menos de 6,5 libras), ajustado por sexo y año de nacimiento, mostraron un riesgo mayor de diabetes comparados con el grupo que se tomó como referencia (nacidos con peso entre 6,5 y 8,4 libras) ( $R R=2,10$; IC95\% 1,29-3,41) (18).

En China, Li y cols (2010), estudiaron una cohorte de 7.874 personas nacidas entre octubre de 1952 y septiembre de 1964 residentes en áreas rurales del país, contaron con un grupo expuesto de $1.005(12,8 \%)$ a "hambruna fetal", otro grupo de 4.915 expuestos a una temporada de hambruna durante la niñez y un grupo control no expuesto de 1.954 (24,8\%); observaron que el grupo de expuestos durante la vida fetal y la niñez a la hambruna mostraron un riesgo incrementado para diabetes adulta, ajustado por otras variables (sexo, nivel educativo, historia familiar de diabetes, consumo actual de cigarrillo y alcohol y nivel de actividad física). La prevalencia de diabetes en el grupo con exposición fetal fue $3,5 \%$; en la niñez temprana $1,2 \%$; niñez mediana $2,9 \%$; niñez tardía $2,0 \%$ y en el grupo

\section{Tabla I \\ ASOCIACIONES ENTRE RESTRICCIÓN DEL CRECIMIENTO INTRAUTERINO $Y$ ENFERMEDADES EN LA VIDA ADULTA}

\begin{tabular}{lcc}
\hline Autores & Desenlace & OR/RR/HR (IC95\%) \\
\hline Zhao y cols (17) & Hipertensión arterial & $0,95(0,81-1,11)^{\star \star}$ \\
Burke y cols (18) & Diabetes & $2,10(1,29-3,41)$ \\
Painter y cols (19) & Enfermedad coronaria & $1,90(1,00-3,80)^{\star}$ \\
de Rooij y cols (20) & Síndrome metabólico & $1,29(1,00-1,68)^{\star \star}$ \\
Li y cols (21) & Diabetes & \\
& Fetal & $1,43(0,53-3,87)$ \\
& Niñez temprana & $2,07(0,82-5,24)$ \\
& Niñez mediana & $0,75(0,26-2,12)$ \\
van Abeelen y cols (22) & Niñez tardía & $2,51(0,91-6,87)$ \\
& Diabetes & $1,36(1,09-1,70)^{\star}$ \\
Wang y cols (23) & Hambruna moderada & $1,64(1,26-2,14)^{\star}$ \\
& Hambruna severa & $1,03(0,71-1,49)$
\end{tabular}

HR, Razón de probabilidad. * Se informa HR. ** Se calculó el RR, no ajustado. 
control 1,4\%. La comparación de cada grupo con el grupo no expuesto mostró falta de relación entre la exposición y el diagnóstico de diabetes en la vida adulta $(R R=1,43$; IC95\% 0,53-3,87 para la exposición fetal; $R R=2,07 ; \mathrm{IC} 95 \% 0,82-5,24$ para la niñez temprana; RR=0,75; IC95\% 0,26-2,12 y $R R=2,51$; IC95\% 0,91-6,87 para la niñez tardía) (21).

En Holanda, Abeelen y cols (2012) siguieron una cohorte de 7.837 mujeres entre 49 y 70 años expuestas y no expuestas a un período de hambruna durante seis meses después de terminada la Segunda Guerra Mundial en las áreas urbana. Contaron con un grupo control de 3.572 mujeres $(45,6 \%)$, un grupo expuesto a hambruna moderada de 2.975 mujeres $(38,0 \%)$ y otro expuesto a hambruna severa de $1.290(16,4 \%)$ y observaron una prevalencia de diabetes del $4,0 \%$ en el grupo control, $5,8 \%$ en el grupo expuesto a hambruna moderada y $7,0 \%$ en las expuestas hambruna severa. En la comparación con el grupo control incluyeron el tiempo de exposición y observaron que la historia de hambruna moderada se asoció a inicio más temprano de diabetes, ajustado por edad ( $\mathrm{HR}=1,36$; IC95\% 1,09-1,70) y mujeres con exposición severa, ajustado por edad (HR=1,64; IC95\% 1,26-2,14) (22).

\section{HIPERTENSIÓN}

En China, Zhao y cols (2002) en una cohorte en el que participaron 13.467 mujeres entre $40 \mathrm{y}$ 70 años de Shanghai, tomaron como exposición el bajo peso al nacer, definido como el peso inferior a 2.500 gramos y se observó que este no representó factor de riesgo estadísticamente significativo para la presentación de hipertensión arterial en la adultez (mayores de 40 años). Dado que el artículo no presentó esta información se calculó el valor crudo (RR=0,95; IC95\% 0,81-1,11) (17).

También en China, Wang y cols (2012) en una cohorte de 12.065 personas, hombres y mujeres en edades entre 46 y 43 años, nacido entre 1957 y 1964 en Zhongshan y Nanhai y que estuvieron o no expuestos al período de hambruna más largo de la historia de humanidad (1959 y 1961) informaron que la exposición no guardó asociación estadística con hipertensión arterial en la adultez, ajustada por variables demográficas y estilo de vida $(R R=1,03$; IC95\% 0,71-1,49) (23).

\section{ENFERMEDAD CORONARIA}

En Holanda, Painter y cols (2006) analizaron una cohorte de 2.414 participantes divididos en cinco grupos (previo a la hambruna, gestación temprana, gestación media, gestación tardía y posterior a la hambruna). La exposición consistió en el evento de un período no menor de 13 semanas con una ingesta adulta inferior a 1.000 calorías por día.
Se halló que las personas en los grupos expuestos presentaron más tempranamente enfermedad coronaria que las no expuestas a la hambruna, se incluyó en el análisis el tiempo de exposición (HR=1,9; IC95\% 1,0-3,8) (19).

\section{OBESIDAD}

En China, Wang y cols (2012) siguieron cohortes con exposición y no exposición a hambruna antes descrita y realizaron un análisis estratificado por sexo y observaron tanto en hombres $(R R=1,20$; IC95\% 0,63-2,29) como en mujeres ( $R R=1,07$; IC95\% 0,53-2,14) que la exposición a la hambruna no incrementó estadísticamente el riesgo de obesidad (23).

\section{SÍNDROME METABÓLICO}

En Holanda, de Rooij y cols (2007) siguieron por varias décadas una cohorte de 2.414 personas con exposición o no a hambruna (la misma de un informe antes anotado) y se halló una asociación estadísticamente significativa entre la exposición y el desenlace síndrome metabólico; dado que el artículo omitió la presentación del estimador se calculó el valor crudo (RR=1,29; IC95\%1,00-1,68) (20).

\section{DISCUSIÓN}

En la presente revisión se observa que un número reducido de investigaciones informan la asociación entre RCIU y los desenlaces analizados en la vida. Esto no permite un análisis concluyente.

Aunque, algunas investigaciones muestran una asociación significativa entre RCIU y cambios negativos en algunos parámetros metabólicos como tolerancia a la glucosa, adiposidad o niveles séricos de lípidos, medidos como variables cuantitativas, es muy reducida la información sobre desenlaces categóricos, como condiciones clínicamente definidas como diabetes o hipertensión (15,24-36). Igualmente, los estudios muestran alta heterogeneidad, no sólo en las características de las poblaciones participantes sino también en la definición de las exposiciones y desenlaces evaluados (17-23).

Sin duda, la teoría de Barker es una propuesta interesante para explicar en parte la compleja interacción entre factores genéticos y ambientales como predisponentes en la aparición de enfermedades $(1,2)$. No obstante, la observación de la frecuencia de RCIU como factor predisponente, con cifras hasta de $15 \%$ (4) y de la diabetes (40\%), hipertensión arterial $(60 \%)$ y obesidad (30) sugieren que los problemas nutricionales en la vida intrauterina, y posiblemente en los primeros años de la infancia, explican un porcentaje menor de los casos que se observan en la adultez (10-14). 
Durante mucho tiempo se consideró que desarollo fetal se encontraba completamente aislado de factores externos potencialmente nocivos; sin embargo, la relación observada entre el desarrollo fetal y las enfermedades de aparición en la adultez refutan esa teoría y por lo tanto se plantearon otras hipótesis interrelacionadas para explicarla: genotipo ahorrativo (thrifty genotype), genotipo de bebé pequeño sobreviviente (survivor small baby genotype), insulina fetal (fetal insuline), fenotipo ahorrativo (thrifty phenotype), orígenes fetales (fetal origens), exposición a glucocorticoides fetales (fetal glucocorticoid exposure), programación (programming) y recuperación de crecimiento (catch-up growth) (41-57).

Se plantea que los recién nacidos con restricción nutricional presentan niveles menores de insulina y factores de crecimiento similares a la insulina como (IGF-1) e IGF unido a la proteína 3 (IGFBP-3); aunque, los niveles de estas hormonas se normalizan generalmente durante los tres primeros meses posnatales, esto coincide con el rápido crecimiento en estos neonatos, es posible que los tejidos expuestos a deficiencia crónica de insulina y de IGF-1 desarrollen resistencia a la insulina como mecanismo de defensa hacia la hipoglicemia y con ello se incremente el riesgo de síndrome metabólico y obesidad posnatal $(41,42)$.

Igualmente, se propone que la pobre nutrición en la vida temprana produce alteraciones en órganos como el páncreas, con posterior insulinorresistencia, riñones e hígado, que predispone a hipertensión arterial y diabetes mellitus, mientras se protegen órganos como cerebro, en un proceso de adaptación metabólica $(42,43)$.

Asimismo, se considera que la carga y expresión génica no son rígidas, sino que interactúan de diversos factores ambientales que actúan en todo el desarrollo para establecer un fenotipo definido (44). Es posible que esta imbricación de factores genéticos y ambientales induzcan un proceso de "reprogramación metabólica" y de selección que favorezca la supervivencia de los predispuestos genéticamente, a costa de una disfunción mitocondrial con cambios cuantitativos en el contenido del ácido desoxirribonucleico mitocondrial (ADNmt), planteados como una anormalidad importante en la insulinorresistencia y la diabetes mellitus tipo 2 $(45,46)$.

La hipótesis de programación fetal propone que la enfermedad coronaria, el accidente cerebrovascular, la HTA y la diabetes mellitus tipo 2 se originan en la plasticidad del desarrollo que ocurre en respuesta a factores maternos y placentarios durante la vida fetal y la lactancia. Un segundo proceso comprende la regulación hormonal y metabólica. Un recién nacido prematuro o con BPN presenta mayor susceptibilidad de tener un patrón metabólico "ahorrativo", con un crecimiento acelerado en el período posnatal, para el manejo de los nutrientes relacionado con una reducción en el número de células pancreáticas a la que se le suma una resistencia periférica a la insulina $(47,48)$. $Y$ tercero, la programación fetal hace más vulnerables a las influencias ambientales adversas en la adultez $(49,50)$.

Otras alteraciones relacionadas con la restricción nutricional materna incluyen cambios en el sistema muscular como un incremento de triglicéridos intramusculares (IMTG) que explica parcialmente la predisposición a diabetes mellitus tipo 2. Los altos niveles de IMTG se asocian a reducción de la actividad de la enzima carnitina palmitoil-1 (CPT1b), una proteína clave en la oxidación de estos ácidos grasos (41). Asimismo, se observa disminución de las fibras de tipo I y lla en sujetos con bajo peso al nacer que se acompaña de una marcada reducción en la capacidad oxidativa muscular, situación que se asocia a resistencia a la insulina y obesidad en la adultez. A comienzos del desarrollo fetal es la mayor expresión de fibras musculares tipo I, mientras que las fibras tipo Ila, Ilb y IIx se desarrollan en etapas más tardías $(51,52)$. Por lo tanto, sería razonable formular la hipótesis que el músculo esquelético juega un papel clave en la programación prenatal (53). La descendencia de las madres que recibieron una dieta con restricción de nutrientes y calorías durante el último trimestre de embarazo presentaron una disminución en el número de fibras musculares y un aumento relativo en el número de fibras tipo Ilb en comparación con los hijos alimentados de madres con adecuado aporte nutrientes (54).

Asimismo, una buena placentación y, en consecuencia, un adecuado desarrollo fetal guarda relación con la presencia de hormonas como leptina y adiponectina (55). Los niños de madres que presentan disminución en niveles de adiponectina es similar a los neonatos con RCIU. Sin embargo, estos niños presentan niveles aumentados de leptina al compararlos con niños con peso adecuado al nacer un año después del nacimiento. Finalmente, adultos con historia de bajo peso al nacer muestran incremento en niveles de leptina al compararse con personas con igual índice de masa corporal $(55,56)$.

Otros investigadores informan cambios renales (reducción del número de nefronas), neuroendocrinos (desregulación del eje hipotálamo-pituitariaadrenal), y vasculares (disfunción vascular endotelial, propiedades angiogénicas endoteliales de las células formadoras de colonias, y reducción de la densidad de las arteriolas y capilares) (57).

La interacción entre componentes genéticos y el crecimiento desde la vida intrauterina puede permitir el diseño de intervenciones de promoción y prevención individuales y colectivas para los grupos en alto riesgo de enfermedades que adquieren la condición de cronicidad en la adultez (58). Se 
acepta que el efecto de RCIU sobre los resultados perinatales y el neurodesarrollo en la infancia guarda una relación inversa con la edad gestacional al momento de la exposición y el percentil de crecimiento al nacimiento (5). En la actualidad, se recomienda la valoración del flujo de la arteria umbilical con Doppler en embarazos con RCIU $(4,58)$. Un adecuado seguimiento reduce la necesidad de inducción del parto, las cesáreas y muertes perinatales. Sin embargo, el estudio Doppler de la arteria umbilical es pobre predictor de eventos cardiovasculares en la adultez (59).

Esta revisión compila la evidencia disponible de estudios de casos y controles y de cohortes que muestran las implicaciones a largo plazo de RCIU. Sin embargo, tiene la limitación que incluyó pocas investigaciones en los que se midieron exposición y desenlaces de forma disímil lo que limita la evaluación en conjunto. Estas divergencias inducen sesgos, hacen difíciles las comparaciones y llegar a conclusiones válidas. No obstante, es un aporte para comprensión del fenómeno y pensar en la necesidad de nuevas o integración de teorías en un modelo más amplio para explicar la complejidad de la asociación dado que, igualmente, RCIU se ha relación con la presentación de trastornos mentales, como la esquizofrenia (60).

\section{CONCLUSIONES}

Se concluye que RCIU muestra una relación inconsistente con enfermedades adultas en estudios observacionales. Se necesitan estudios que aclaren la asociación entre RCIU y enfermedades en la vida adulta observada. Estas investigaciones se deben realizar con poblaciones de los cinco continentes y de esta manera reunir la diversidad genética y medioambiental mundial.

\section{REFERENCIAS}

1. Barker DJ, Osmond C, Winter PD, Margetts B, Simmonds SJ. Weight in infancy and death from ischaemic heart disease. Lancet 1989;334:577-80.

2. Barker D, Osmond C, Golding J, Kuh D, Wadsworth ME. Growth in utero, blood pressure in childhood and adult life, and mortality from cardiovascular disease. BMJ 1989;298:564-7.

3. Miller J, Turan S, Baschat AA. Fetal growth restriction. Semin Perinatal 2008;32:274-80.

4. Sanín-Blair JE, Díaz JG, Ramírez J, Mejía CA, Medina Ó, Vélez J. Diagnóstico y seguimiento del feto con restricción del crecimiento intrauterino (RCIU) y del feto pequeño para la edad gestacional (PEG). Consenso colombiano. Rev Colomb Obstetr Ginecol 2009;60:247-61.

5. Durán P. Nutrición temprana y enfermedades en la edad adulta: acerca de la "hipótesis de Barker". Arch Arg Pediatr 2004;102:26-34.
6. Osmond C, Barker DJ. Fetal, infant, and childhood growth are predictors of coronary heart disease, diabetes, and hypertension in adult men and women. Environ Health Perspect 2000;108 (Suppl. 3):545-53.

7. Gillman MW. Developmental origins of health and disease. N Engl J Med 2005;353:1848-50.

8. Lumey LH, Stein AD, Susser E. Prenatal famine and adult health. Ann Rev Public Health 2011;32:237-62.

9. Negrato CA, Gomes MB. Low birth weight: causes and consequences. Diabetol Metab Syndr 2013;5:49. Retraction in: Diabetol Metab Syndr 2014:6:60.

10. Narayan KV, Boyle JP, Thompson TJ, Sorensen SW, Williamson DF. Lifetime risk for diabetes mellitus in the United States. JAMA 2003;290:1884-90.

11. Kearney PM, Whelton M, Reynolds K, Whelton PK, $\mathrm{He} J$. Worldwide prevalence of hypertension: a systematic review. J Hypertension 2004;22:11-9.

12. Berghöfer A, Pischon T, Reinhold T, Apovian CM, Sharma AM, Willich SN. Obesity prevalence from a European perspective: a systematic review. BMC Public Health 2008;8:200.

13. Flegal KM, Carroll MD, Ogden CL, Curtin LR. Prevalence and trends in obesity among US adults, 19992008. JAMA 2010;303:235-41.

14. Alhyas L, McKay A, Majeed A. Prevalence of type 2 diabetes in the States of the co-operation council for the Arab States of the Gulf: a systematic review. PloS One 2012;7:e40948.

15. Whincup PH, Kaye SJ, Owen CG, Huxley R, Cook $\mathrm{DG}$, Anazawa, S, et al. Birth weight and risk of type 2 diabetes: a systematic review. JAMA 2008;300:288697.

16. Mousa HA, Loughna P. Fetal growth restriction: investigation and treatment. Obstet Gynaecol Reprod Med 2008;18:247-52.

17. Zhao M, Shu XO, Jin F, Yang G, Li HL, Liu DK, et al. Birthweight, childhood growth and hypertension in adulthood. Int J Epidemiol 2002;31:1043-51.

18. Burke JP, Forsgren J, Palumbo PJ, Bailey KR, Desai $\mathrm{J}$, Devlin $\mathrm{H}$, et al. Association of birth weight and type 2 diabetes in Rochester, Minnesota. Diabetes Care 2004;27:2512-3.

19. Painter RC, de Rooij SR, Bossuyt PM, Simmers TA, Osmond C, Barker DJ, et al. Early onset of coronary artery disease after prenatal exposure to the Dutch famine. Am J Clin Nutr 2006;84:322-7.

20. de Rooij SR, Painter RC, Holleman F, Bossuyt PM, Roseboom TJ. The metabolic syndrome in adults prenatally exposed to the Dutch famine. Am J Clin Nutr 2007;86:1219-24.

21. Li Y, He Y, Qi L, Jaddoe VW, Feskens EJ, Yang X, et al. Exposure to the Chinese famine in early life and the risk of hyperglycemia and type 2 diabetes in adulthood. Diabetes 2010;59:2400-6.

22. van Abeelen AF, Elias SG, Bossuyt PM, Grobbee DE, van der Schouw YT, Roseboom TJ, et al. Famine exposure in the young and the risk of type 2 diabetes in adulthood. Diabetes 2012 61:2255-60.

23. Wang PX, Wang JJ, Lei YX, Xiao L, Luo ZC. Impact 
of fetal and infant exposure to the Chinese great famine on the risk of hypertension in adulthood. Plos One 2012;7:e49720.

24. Barker DJ. Fetal origins of coronary heart disease. BMJ 1995;311:171-4.

25. Martyn CN, Barker DJ, Jespersen S, Greenwald S, Osmond C, Berry C. Growth in utero, adult blood pressure, and arterial compliance. Br Heart J 1995;73:11621.

26. Leon DA, Koupilova I, Lithell HO, Berglund L, Mohsen $\mathrm{R}$, Vagero $\mathrm{D}$, et al. Failure to realize growth potential in utero and adult obesity in relation to blood pressure in 50 year old Swedish men. BMJ 1996;312:401-4.

27. Roseboom TJ, van der Meulen JH, Ravelli $A C$, van Montfrans GA, Osmond C, Barker DJ, et al. Blood pressure in adults after prenatal exposure to famine. $\mathrm{J}$ Hypertension 1999;17:325-30.

28. Levitt NS, Lambert EV, Woods D, Hales CN, Andrew $\mathrm{R}$, Seckl JR. Impaired glucose tolerance and elevated blood pressure in low birth weight, nonobese, young South African adults: Early programming of cortisol axis 1. J Clin Endocrinol Metab 2000;85:4611-8.

29. Krishnaswamy K, Naidu AN, Prasad MPR, Reddy GA. Fetal malnutrition and adult chronic disease. Nutr Rev 2002;60:S35-S39.

30. Barker DJ, Eriksson JG, Forsen T, Osmond C. Fetal origins of adult disease: strength of effects and biological basis. Int J Epidemiol 2002;31:1235-9.

31. Dempsey JC, Williams MA, Luthy DA, Emanuel I, Shy $\mathrm{K}$. Weight at birth and subsequent risk of preeclampsia as an adult. Am J Obstet Gynecol 2003;189:494500.

32. Tuya C, Mutch WJ, Haggarty P, Campbell DM, Cumming A, Kelly K, et al. The influence of birth weight and genetic factors on lipid levels: a study in adult twins. $\mathrm{Br}$ J Nutr 2006;95:504-10.

33. Stein AD, Kahn HS, Rundle A, Zybert PA, van der Pal-de Bruin K, Lumey LH. Anthropometric measures in middle age after exposure to famine during gestation: evidence from the Dutch famine. Am J Clin Nutr 2007;85:869-76.

34. Lumey LH, Stein AD, Kahn HS, van der Pal-de KM, Blauw GJ, Zybert PA, et al. Cohort profile: the Dutch Hunger Winter families study. Int $\mathrm{J}$ Epidemiol 2007;36:1196-204.

35. Hovi $P$, Andersson $S$, Eriksson JG, Järvenpää AL, Strang-Karlsson S, Mäkitie $\mathrm{O}$, et al. Glucose regulation in young adults with very low birth weight. N Engl J Med 2007;356:2053-63.

36. Thomas N, Grunnet LG, Poulsen P, Christopher S, Spurgeon R, Inbakumari M, et al. Born with low birth weight in rural Southern India: what are the metabolic consequences 20 years later? Eur J Endocrinol 2012;166: 647-55.

37. Hofman PL, Regan F, Jackson WE, Jefferies C, Knight DB, Robinson EM, et al. Premature birth and later insulin resistance. N Engl J Med 2004;351:217986.
38. Vohr BR, Allan W, Katz KH, Schneider KC, Ment LR. Early predictors of hypertension in prematurely born adolescents. Acta Paediatr 2010;99:1812-8.

39. Hack M, Schluchter M, Andreias L, Margevicius S, Taylor HG, Drotar D, et al. Change in prevalence of chronic conditions between childhood and adolescence among extremely low-birth-weight children. JAMA 2011;306:394-401.

40. Hill DJ, Prapavessis H, Shoemaker JK, Jackman M, Mahmud $\mathrm{FH}$, Clarson C. Relationship between birth weight and metabolic status in obese adolescents. ISRN Obesity 2013. Doi: 10.1155/2013/490923.

41. Taniguchi CM, Emanuelli B, Kahn CR. Critical nodes in signalling pathways: insights into insulin action. Nat Rev Mol Cell Biol 2006;7:85-96.

42. Cianfarani S, Germani D, Branca F. Low birthweight and adult insulin resistance: the catch-up growth hypothesis. Arch Dis Child Fetal Neonatal Ed 1999;81:713.

43. Fowden AL. The role of insulin in prenatal growth. $J$ Dev Physiol 1989;12:173-82.

44. Sebert S, Sharkey D, Budge H, Symonds ME. The early programming of metabolic health: is epigenetic setting the missing link? Am J Clin Nutr 201194 (Suppl. 6):S1953-S1958.

45. Song J, Oh JY, Sung YA, Pak YK, Park KS, Lee HK. Peripheral blood mitochondrial DNA content is related to insulin sensitivity in offspring of type 2 diabetic patients. Diabetes Care 2001;24: 865-89.

46. Gemma C, Sookoian S, Alvarinas J y cols. Mito $\neg$ chondrial DNA depletion in small- and large-forgesta $\neg$ tional-age newborns. Obesity 2006;14:2193-9.

47. Baker JL, Olsen LW, Sorensen TIA. Childhood bodymass index and the risk of coronary heart disease in adulthood. N Engl J Med 2007;357:2329-33.

48. Fernandez-Twinn DS, Ozanne SE. Mechanisms by which poor early growth programs type-2 diabetes, obesity and the metabolic syndrome. Physiol Behav 2006;88:234-43.

49. Lau C, Rogers JM, Desai M, Ross MG. Fetal programming of adult disease: Implications for prenatal care. Obstet Gynecol 2011;117:978-85.

50. Boney CM, Verma A, Tucker R, Vohr BR. Metabolic syndrome in childhood: association with birth weight, maternal obesity, and gestational diabetes mellitus. Pediatrics 2005;115:e290-e296.

51. Tilley RE, McNeil CJ, Ashworth CJ, Page KR, Mcardle HJ. Altered muscle development and expression of the insulin-like growth factor system in growth retarded fetal pigs. Domest Anim Endocrinol 2007;32:167-77.

52. Malenfant $P$, Joanisse DR, Theriault R, Goodpaster $\mathrm{BH}$, Kelley DE, Simoneau JA. Fat content in individual muscle fibers of lean and obese subjects. Int $\mathrm{J}$ Obes Relat Metab Disord 2001;25:1316-21.

53. Schuler M, Ali F, Chambon C. PGC1alpha expression is controlled in skeletal muscles by PPARbeta, whose ablation results in fiber-type switching, obesity, and type 2 diabetes. Cell Metab 2006;4:407-14. 
54. Zhu MJ, Ford SP, Means WJ, Hess BW, Nathanielsz PW, Du M. Maternal nutrient restriction affects properties of skeletal muscle in offspring. J Physiol 2006;575:241-50.

55. Gluckman PD, Hanson MA. Living with the past: evolution, development, and patterns of disease. Science 2004;305:1733-6.

56. Wang J, Shang LX, Dong X, Wang X, Wu N, Wang $\mathrm{SH}$, et al. Relationship of adiponectin and resistin levels in umbilical serum, maternal serum and placenta with neonatal birth weight. Aust N Z J Obstet Gynaecol 2010;50:432-8.
57. Hanson M, Gluckman P. Endothelial dysfunction and cardiovascular disease: the role of predictive adaptive responses. Heart 2005;91:864-6.

58. Eriksson JG. Epidemiology, genes and the environment: lessons learned from the Helsinki Birth Cohort Study. J Intern Med 2007;261:418-25.

59. Hui L, Challis D. Diagnosis and management of fetal growth restriction: the role of fetal therapy. Best Pract Res Clin Obstetr Gynaecol 2008;22:139-58.

60. Freedman D, Bao Y, Kremen WS, Vinogradov S, McKeague IW, Brown AS. Birth weight and neurocognition in schizophrenia spectrum disorders. Schizophrenia Bull 2013;39:592-600. 